\title{
System-Level Modelling of Surface Acoustic Wave Strain Sensors
}

\author{
Brian Mc Cormack ${ }^{\dagger}$, Dermot Geraghty ${ }^{\dagger}$ and Margaret O'Mahony* \\ Centre for Transport Research and Innovation for People (TRIP) \\ ${ }^{\dagger}$ Department of Mechanical and Manufacturing Engineering \\ ${ }^{*}$ Department of Civil, Structural and Environmental Engineering \\ Trinity College Dublin \\ IRELAND \\ E-mail: ${ }^{\dagger}$ mecormb@tcd.ie
}

\begin{abstract}
Surface Acoustic Wave (SAW) devices may be configured as strain sensors, providing passive, wireless strain measurement in demanding conditions, A key consideration is the modelling of the sensors, enabling different device designs to be considered. These simulations may then be used in system-level models of complete measurement systems. This paper presents a simulation scheme using Coupling-Of-Modes (COM) analysis. Preliminary results are compared to experimental values from the literature, and good agreement is found.

Keywords - SAW, strain, modelling.
\end{abstract}

\section{INTRODUCTION}

Surface Acoustic Wave (SAW) devices have been used for many years in RF communications as filters or as part of oscillator circuits. In recent years, their potential as wireless sensors has begun to be exploited [1]. The key advantage of SAW sensors is that they can provide passive, wireless measurement in a wide variety of applications.

An important issue with SAW sensors is the modelling of sensor response to the measurand (and to other influences). This allows the sensor performance to be evaluated at the design stage and the comparison of different design configurations. SAW devices are increasingly being used in multi-sensor systems, and accurate modelling can be used to investigate sensor interference and other effects. The modelling of strain effects on SAW sensors has been explored in [2] and [3], but most of the other work on these sensors has been experimental.

This paper proposes the use of Coupling-OfModes (COM) analysis [4] to model the effect of strain on device behaviour. COM analysis is frequently used in the design of SAW devices for communications, but it may be extended to sensor simulation. The operation of a SAW strain sensor is described, and the COM formulation with strain effects is introduced. Preliminary results are compared to experimental values [2], and good agreement is found.

\section{SAW Strain Sensors}

The devices to be modelled are 1-port SAW resonators (SAWR), illustrated in Figure 1. These consist of thin film electrodes deposited on a piezoelectric substrate. An interdigital transducer (IDT) is used to convert an RF interrogation signal (which may be transmitted wirelessly) into propagating surface waves by the inverse piezoelectric effect. The wave energy is concentrated near the surface of the substrate, enabling easy transduction and reflection. 1-port SAW resonators have a reflector grating on each side of the IDT to reflect most of the incident SAW energy back to the source. Thus the device resonates at a frequency determined by the geometry of the electrode structures and the material properties of the substrate [5].

If the substrate is strained by an external load (e.g., in the direction of SAW propagation as shown in Figure 1), the geometry of the electrode structure and the propagation properties of the substrate are changed: this causes the resonant frequency to shift. By measuring this shift in resonant frequency, the applied strain on the device may be estimated. For the loading case shown in Figure 1, a uniaxial strain sensor (sensitive in the direction of SAW propagation) is thus created. The aim of this paper is to show how this frequency shift may be modelled, and the parameters which need to be considered. 


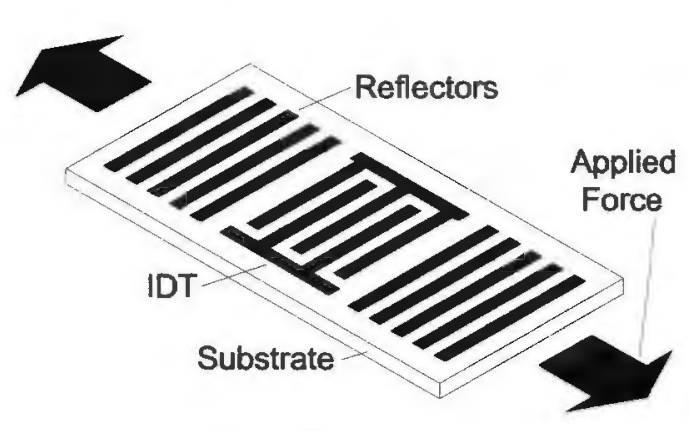

Fig. 1: SAWR under strain

\section{Modelding LeVels}

In a multi-sensor environment, two different modelling levels may be considered: device level and system level. In device level modelling, the sensor is treated as an electro-mechanical body, i.e., a composite structure consisting of a piezoelectric substrate with deposited metallic electrodes and reflectors, subject to mechanical and electrical loads. Given the complexity of the structures and materials, Finite or Boundary Element schemes are usually required to investigate device behaviour. While this approach may provide good insight into sensor performance under external load, the processing requirements are such that all but the simplest analyses are unfeasible.

System level modelling may also be employed. In this case the sensor is treated as a small part of a larger measurement system, effectively a 'black box' which is subject to system signals and external loads. A basic version is shown in Figure 2. The interrogating signal is produced by the interrogator model (with attendant channel models if required) and applied to the sensor block. Independent inputs (e.g., applied strain, temperature) are also applied, and the response signal is evaluated. This approach has general application, and allows for easy integration with various interrogator, channel and antenna models. A key disadvantage, however, is that no direct information is provided on the strain state of the device, as the sensor model usually consists of an equivalent circuit with lumped component values [6].

In this work, a core system level model (using the COM approach) is augmented with device level modelling of strain effects. These effects are included in the the independent COM parameters, and are described in the next section.

\section{COM Modelling}

COM analysis may be used to model wave propagation in many types of periodic media [7]. It

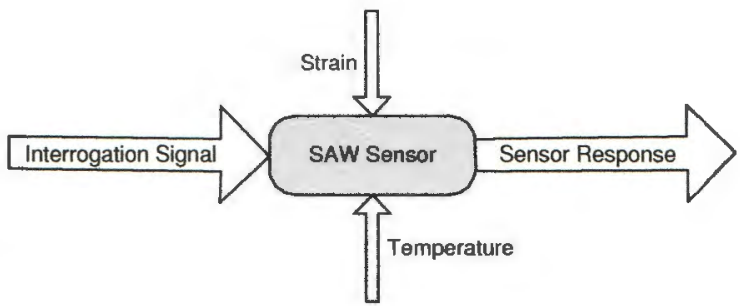

Fig. 2: System level model of SAW sensor

describes the interaction between incident and reflected waves in a perturbed region: for SAW devices this coupling occurs in the IDT and reflector grating structures. In a narrow frequency range around the nominal centre frequency of a SAW structure (IDT or reflector), the infinite wave fields in the structure at position $x$ may be approximated by [4]:

$$
\begin{aligned}
& \frac{\mathrm{d} R(x)}{\mathrm{d} x}=-\mathrm{i} \delta R(x)+\mathrm{i} \kappa S(x)+\mathrm{i} \alpha V \\
& \frac{\mathrm{d} S(x)}{\mathrm{d} x}=-\mathrm{i} \kappa^{*} R(x)+\mathrm{i} \delta S(x)-\mathrm{i} \alpha^{*} V \\
& \frac{\mathrm{d} I(x)}{\mathrm{d} x}=-2 \mathrm{i} \alpha^{*} R(x)-2 \mathrm{i} \alpha S(x)+\mathrm{i} \omega C V
\end{aligned}
$$

In the coupled equations above, $R(x)$ and $S(x)$ are the slowly-varying wave fields, $I(x)$ is the IDT current, $\delta=2 \pi\left(f-f_{0}\right) / v-\mathrm{i} \gamma$ is the detuning parameter, $v$ is the SAW velocity, $\gamma$ is the SAW attenuation, $\kappa$ is the structure reflectivity, $\alpha$ is the transduction parameter, $\omega=2 \pi f$ is the wave frequency, $C$ is the capacitance and $V$ is the applied voltage; complex conjugation is denoted by an asterisk. $R(x)$ and $S(x)$ are two eigenmodes which represent the forward- and backward-propagating waves at a given position in the SAW device structure. Electrode resistance has not been included here for simplicity, but can be included if required. COM expressions for each structure in a SAW device may be linked using P-matrices [8] to model the overall frequency response.

Figure 3 shows the calculated admittance response of a synchronous 1-port SAWR on ST-X quartz around its resonant frequency. The device has 60 finger pairs in the IDT and 650 electrodes per reflector grating; each finger is $1.82 \mu \mathrm{m}$ wide and $150 \mathrm{~nm}$ thick. The resonance occurs at the low-frequency edge of the stopband, and is caused by constructive interference of the counterpropagating waves. At the high-frequency edge of the stopband the interference is destructive, hence the absence of a conductance peak. This form of detailed frequency response is crucial in multisensor networks where device responses may interfere. COM analysis allows this these effects to be modelled in a much more intuitive way than other 


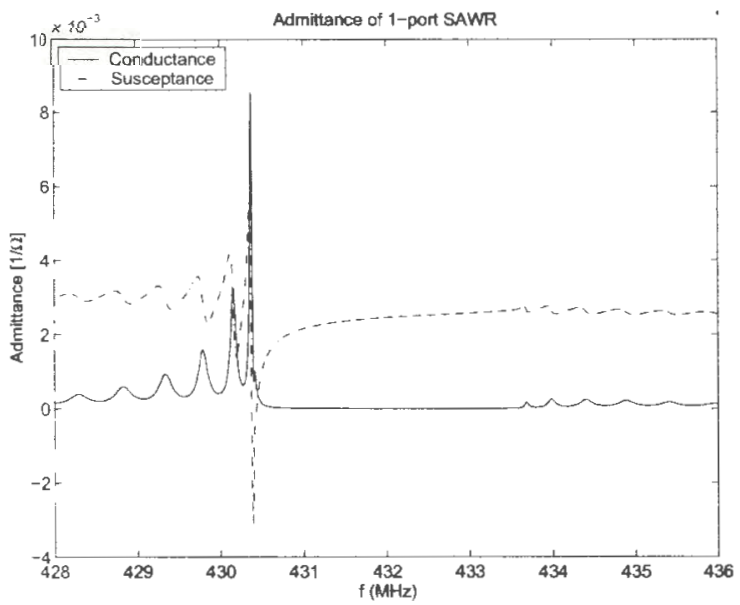

Fig. 3: COM admittance of 1-port SAWR

simulation schemes such as equivalent circuits, as propagating waves rather than lumped circuit elements are modelled.

A major advantage of the COM formulation is that device dimensions are explicitly defined in the model and thus they can be made strain-sensitive. The width, thickness and length of each electrode is defined in the model, as well as the number of electrodes in the structure. If the device is strained as shown in Figure 1, we may define the strained electrode dimensions as:

$$
\begin{gathered}
w=w_{0}(1+\varepsilon) \\
h=h_{0}(1-\nu \varepsilon)
\end{gathered}
$$

Here $w$ and $h$ are the electrode width and height, $\varepsilon$ is the applied uniaxial strain and $\nu$ is Poisson's ratio for the electrode material (usually isotropic $\mathrm{Al})$.

The independent COM parameters (i.e. $v, \gamma, \kappa$, $\alpha$ and $C$ ) can also be defined as functions of strain. The capacitance of the device causes only a DC shift in the susceptance curve; similarly, electrode resistance shifts the conductance curve. These quantities do not affect the resonant frequency, and thus only four independent parameters need to be considered here.

Figure 4 shows the SAW velocity on a free surface as a function of propagation direction. The anisotropic nature of the substrate means that the SAW velocity varies considerably with propagation dircction; numerical methods are required to create the velocity profile [9]. In ordinary SAW devices such as filters and oscillators, the strains induced by the propagation of SAW may be considered infinitesimal, and the constitutive piezoelectric equations are linear. By contrast, the finite strains found in SAW strain sensors require nonlinear analysis. This may be included in the velocity

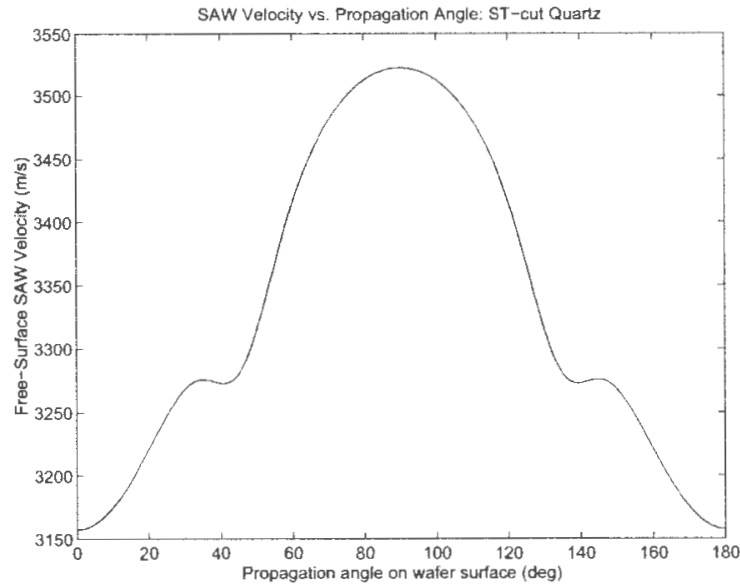

Fig. 4: SAW velocity as a function of propagation angle

determination by modifying the effective material constants of the substrate material and the boundary conditions [10]. These effective constants depend on the stress state of the material, which may be determined analytically for simple loading conditions [11]. More complex loading conditions require the use of the device modelling schemes mentioned above. The unloaded SAW velocity $v_{0}$ plotted in Figure 4 is modified by the presence of the electrodes on the surface of the SAWR [5], and for ST-X quartz the velocity under an electrode structure may be approximated by:

$$
v=v_{0}\left(0.9996-0.02\left(\frac{h}{\lambda}\right)-7.9\left(\frac{h}{\lambda}\right)^{2}\right)
$$

Here $\lambda=4 w$ is the strained SAW wavelength. Figure 5 plots the piezoelectric coupling coefficient as a function of propagation direction. This coefficient determines the coupling between the electrical and mechanical fields in the substrate, and may be estimated from the free and conducting surface SAW velocities [9]. In the COM model the coefficient is proportional to the COM transduction coefficient $\alpha$ [4], and determines the height of the conductance peak.

The SAW attenuation on the free surface of ST$\mathrm{X}$ quartz may be estimated from [12]:

$$
\gamma=\frac{0.47+2.62 \times 10^{-9} f}{8685.9 \lambda}
$$

In (5) $\gamma$ is in Nepers and $f$ is in Hz. This parameter must include all acoustic loss mechanisms in a SAW device [4], and hence the attenuation is larger than that calculated for a free surface. Recent work [13] has proposed more complete attenuation modelling, but at present this parameter is usually measured experimentally. 


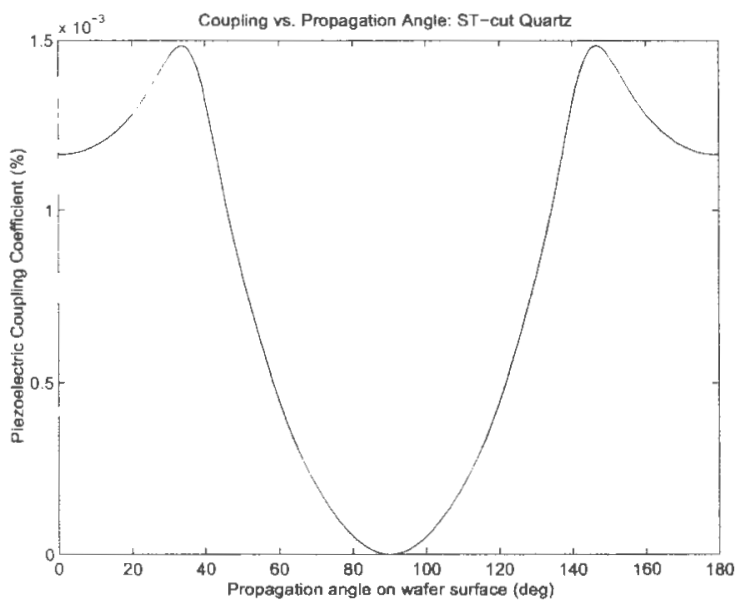

Fig. 5: Coupling as a function of propagation angle

The reflectivity of the structure on ST-X quartz may be expressed as a function of the electrode height and strained wavelength [4]:

$$
\kappa=-\frac{2}{\lambda}\left(0.0004-0.54\left(\frac{h}{\lambda}\right)\right)
$$

It is clear that the definition of reflectivity naturally includes the influence of strain.

\section{Simulation Results}

Figure 6 shows the shift in resonant frequency with an applied flexural strain of -500 to $500 \mu \varepsilon$ on the substrate surface; the device parameters are similar to those described in [2]. The dashed line shows results from experimental SAWR sensors from the same source which have an absolute strain sensitivity of $495 \mathrm{~Hz} / \mu \varepsilon$. The relationship is linear, which is typical of SAW strain sensors [14]. When only the geometric effects are simulated in the COM model (i.e., only the electrode dimensions are varied) the calculated strain sensitivity is $197 \mathrm{~Hz} / \mu \varepsilon$ (dash-dot curve). Previous work on strained SAW devices using bulk substrates has suggested that the effect of strain is dominated by the geometric effects, but that this is heavily dependent on crystal cut [15]. This is confirmed here, as modelling only the geometric effect on ST-X quartz greatly underestimates the strain sensitivity.

When the effect of strain on velocity is also simulated (solid curve), the sensitivity rises to $488 \mathrm{~Hz} / \mu \varepsilon$, which is in excellent agreement with the experimental results.

Although they have not been included in the analysis, temperature effects can also affect SAW strain sensor performance. These can treated in a similar fashion to the strain influences. Thermal expansion of the substrate and electrode structure may be treated as a geometric effect, with appropriate boundary conditions to model the different

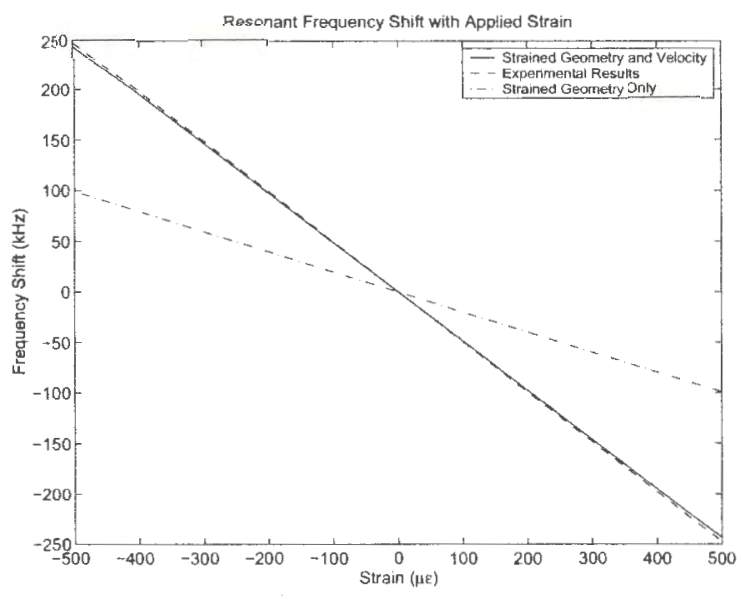

Fig. 6: Resonant frequency shift with applied strain

coefficients of thermal expansion of the sensor and material under test. The propagation properties of the substrate may be altered by using the temperature coefficients of the elastic, piezoelectric and dielectric properties of the quartz substrate [16]. Significant improvements in temperature stability can be achieved though appropriate choice of crystal cut and propagation direction [2].

\section{CONCLUSIONS}

SAW devices can be configured as passive, wireless strain sensors if the substrate is subjected to external loads. The COM formulation provides an excellent model of resonant behaviour, and may be naturally extended to include the effects of strain and other influences. This should provide valuable sensor design information for future SAW measurement systems.

\section{ACKNOWLEDGEMENT}

This work is supported by the HEA under the Programme for Research in Third Level Institutions.

\section{REFERENCES}

[1] A. Pohl, "A review of wireless SAW sensors," IEEE Transactions on Ultrasonics, Ferroelectrics, and Frequency Control, vol. 47, no. 2, pp. $317-332,2000$.

[2] J. Beckley, V. Kalinin, M. Lee, and K. Voliansky, "Non-contact torque sensors based on SAW resonators," in Proc. IEEE International Frequency Control Symposium and PDA Exhibition, 2002, pp. 202 213.

[3] V. Kalinin and A. Leigh, "Influence of nonlinear strain on characteristics of one-port SAW resonators," in Proc. IEEE Ultrasonics Symposium, 2003, pp. 1412-1415. 
[4] V. Plessky and J. Koskela, "Coupling-ofmodes analysis of SAW devices," International Journal of High Speed Electronics and Systems, vol. 10, no. 4, pp. 867-947, 2000.

[5] C. K. Campbell, Surface Acoustic Wave Devices for Mobile and Wireless Communications. San Diego: Academic Press, 1998.

[6] C. C. W. Ruppel, W. Ruile, G. Scholl, K. C. Wagner, and O. Männer, "Review of models for low-loss filter design and applications," in Proc. IEEE Ultrasonics Symposium, 1994, pp. 313-324.

[7] H. Haus and W. Huang, "Coupled-mode theory," Proceedings of the IEEE, vol. 79 , no. 10 , pp. 1505-1518, 1991.

[8] B. P. Abbott, C. S. Hartmann, and D. C. Malocha, "A coupling-of-modes analysis of chirped transducers containing reflective electrode geometries," in Proc. IEEE Ultrasonics Symposium, 1989, pp. 129-134.

[9] A. J. Slobodnik, E. D. Conway, and R. T. Delmonico, Microwave Acoustics Handbook Volume 1A. Massachusetts: USAF Report AFCRL-TR-73-0597, 1973.

[10] B. K. Sinha and H. F. Tiersten, "On the influence of uniaxial biasing stresses on the velocity of piezoelectric surface waves," in Proc. IEEE Ultrasonics Symposium, 1976, pp. 475479.

[11] — , "On the influence of a flexural biasing state on the velocity of piezoelectric surface waves," Wave Motion, vol. 1, pp. 37-51, 1979.

[12] D. P. Morgan, Surface-Wave Devices for Signal Processing. Amsterdan: Elsevier, 1991.

[13] S. Lehtonen, V. P. Plessky, C. S. Hartmann, and M. M. Salomaa, "Extraction of the SAW attenuation parameter in periodic reflecting gratings," IEEE Transactions on Ultrasonics, Ferroelectrics, and Frequency Control, vol. 52, no. 1, pp. 111-119, 2005.

[14] U. T. Zwicker, "Strain sensor with commercial SAWR," Sensors and Actuators, vol. 17, pp. 235-239, 1989.

[15] A. L. Nalamwar and M. Epstein, "Propagation of surface acoustic waves in strained media," in Proc. IEEE Ultrasonics Symposium, 1976, pp. 484-487.

[16] D. F. Nelson, Ed., Low Frequency Properties of Dielectric Crystals (Subvolume $A$ and B). Bcrlin: Landolt-Börnstein, 1993. 